\title{
The Rossby wave extra invariant in the dynamics of 3-D fluid layers and the generation of zonal jets
}

\author{
A. M. Balk \\ Department of Mathematics, University of Utah, Salt Lake City, UT 84112, USA \\ Correspondence to: A. M. Balk (balk@math.utah.edu) \\ Received: 28 February 2013 - Revised: 22 September 2013 - Accepted: 9 November 2013 - Published: 10 January 2014
}

\begin{abstract}
We consider an adiabatic-type (approximate) invariant that was earlier obtained for the quasi-geostrophic equation and the shallow water system; it is an extra invariant, in addition to the standard ones (energy, enstrophy, momentum), and it is based on the Rossby waves. The presence of this invariant implies the energy transfer from small-scale eddies to large-scale zonal jets.

We show that this extra invariant can be extended to the dynamics of a three-dimensional (3-D) fluid layer on the beta plane. Combined with the investigation of other researchers, this 3-D extension implies enhanced generation of zonal jets.

For a general physical system, the presence of an extra invariant (in addition to the energy-momentum and wave action) is extremely rare. We summarize the unique conservation properties of geophysical fluid dynamics (with the beta effect) that allow for the existence of the extra invariant, and argue that its presence in various geophysical systems is a strong indication that the formation of zonal jets is indeed related to the extra invariant.

Also, we develop a new, more direct, way to establish extra invariants (without using cubic corrections). For this, we introduce the small denominator lemma.
\end{abstract}

\section{Introduction}

Alternating zonal jets are found in the large-scale dynamics of oceans and atmospheres under broad conditions. Clearly seen on Jupiter, zonal jets are also unambiguously observed in Earth's oceans (Maximenko et al., 2005). The significance of zonal jets also stems from the well-known mathematical analogy between rotation and magnetic field. Similar equations and similar jets also appear in the dynamics of magnetized plasmas; these jets serve as transport barriers in tokamaks, improving conditions for the controlled nuclear fusion with magnetic confinement (e.g., Manz et al., 2012).
The emergence of zonal jets has been and continues to be intensively studied in both geophysical and plasma contexts. The main purpose of the present paper is to draw attention to the fact that the equations used to describe zonal jets have an unusual adiabatic-like (approximate) invariant (in addition to the usual energy-momentum); this makes the inverse cascade anisotropic and leads to the energy transfer towards zonal jets (see Sect. 4.2). As will be clear below (Sect. 2), this extra invariant is very rare: almost all other physical systems do not have anything analogous to such an invariant. The presence of this conservation in various geophysical systems (within the beta-plane approximation) is a strong indication that the extra invariant indeed plays a role in the emergence of zonal jets.

The extra invariant is adiabatic-like, meaning that the invariant is conserved approximately over a long time. Usually, in the theory of dynamical systems, the adiabatic invariance is due to the slowness of some parameter variation (e.g., Landau and Lifshitz, 1976). However, here the adiabatic-like invariance is due to the smallness of wave amplitudes: it is assumed that the dynamics is weakly nonlinear and is dominated by the triad interactions. It should be noted that the equations describing zonal jets have anisotropy only in linear terms, so strongly nonlinear regimes cannot lead to the zonal-meridional asymmetry. Of course, there are intermediate situations when linear and nonlinear terms have comparable magnitudes. Then real or numerical experiments should be used to see the role of the extra invariant in the formation of zonal jets; we hope that the present paper gives qualitatively the right picture. It is also unknown whether the extra invariant is related to the conservation of potential vorticity, which is an essential feature of geophysical systems. Often, besides weak nonlinearity, we also require the slowness of some parameter variation in space (which usually holds with high accuracy). 
At this point, it is beneficial to recall two seemingly unrelated problems (Sects. 1.1 and 1.2). Then in Sect. 2, we describe the extra invariant and summarize the corresponding unique conservation properties of geophysical systems that allow for its existence. This summary includes the extension of the extra invariant to 3-D fluid layers, which is derived in the present paper (Sect. 3). We see how this invariant implies the energy cascade towards zonal jets in Sect. 4.

\subsection{Boltzmann's collision invariants}

Introducing his kinetic equation, L. Boltzmann (1875) investigated the general form of the thermodynamic equilibrium (Maxwell distribution). Boltzmann studied rarefied gas when the main interaction between the molecules are binary collisions. In each collision, momenta of two molecules before $\left(\boldsymbol{p}_{1}, \boldsymbol{p}_{2}\right)$ and after $\left(\boldsymbol{p}_{3}, \boldsymbol{p}_{4}\right)$ the collision satisfy the momentum-energy conservation:

$p_{1}+p_{2}=p_{3}+p_{4}, \quad p_{1}^{2}+p_{2}^{2}=p_{3}^{2}+p_{4}^{2}$.

These relations imply the conservation of the total momentum and energy

$\boldsymbol{P}=\int \boldsymbol{p} N_{\boldsymbol{p}} \mathrm{d} \boldsymbol{p}, \quad E=\int \boldsymbol{p}^{2} N_{\boldsymbol{p}} \mathrm{d} \boldsymbol{p}$,

where $N_{\boldsymbol{p}}$ is the particle distribution function (the number of molecules with momentum $\boldsymbol{p}$ ). The total number of molecules

$N=\int N_{p} \mathrm{~d} p$

is also conserved, which corresponds to the obvious relation $1+1=1+1$ for each binary collision.

Boltzmann posed the following question: does there exist another function $\varphi(\boldsymbol{p})$ that is also conserved in binary collisions, so that the relations in Eq. (1) imply the relation

$\varphi\left(\boldsymbol{p}_{1}\right)+\varphi\left(\boldsymbol{p}_{2}\right)=\varphi\left(\boldsymbol{p}_{3}\right)+\varphi\left(\boldsymbol{p}_{4}\right) ?$

In other words, does there exist $\varphi(\boldsymbol{p})$, such that Eq. (4) holds for any four vectors $\boldsymbol{p}_{1}, \boldsymbol{p}_{2}, \boldsymbol{p}_{3}, \boldsymbol{p}_{4}$, bound by Eq. (1)? Then Boltzmann's kinetic equation would additionally conserve the integral

$I=\int \varphi(\boldsymbol{p}) N_{\boldsymbol{p}} \mathrm{d} \boldsymbol{p}$

It is supposed that the function $\varphi(\boldsymbol{p})$ is linearly independent of the five functions: 1 , three components of $\boldsymbol{p}$, and $\boldsymbol{p}^{2}$, so that the quantity (5) is independent of quantities (2) and (3).

If there were such a function $\varphi(\boldsymbol{p})$, then the thermodynamic equilibrium would be different. However, Boltzmann (1875) found that there is no such a function $\varphi(\boldsymbol{p})$ (provided it is smooth enough; see also Sercignani, 1990).

\subsection{Zakharov-Schulman test of integrability: degenerative dispersion laws}

In a different development, Zakharov and Schulman (1980, 1988) posed the question "When a given nonlinear wave system is integrable?" They argued that, to be integrable, the system should possess an additional conservation law, besides the energy and the momentum (and, sometimes, the wave action).

Suppose our nonlinear wave system is given in the Fourier representation as dynamics of wave amplitudes $a_{k}(t)$

$$
\begin{aligned}
i \frac{\partial}{\partial t} a_{\boldsymbol{k}_{1}}= & \omega\left(\boldsymbol{k}_{1}\right) a_{\boldsymbol{k}_{1}}+\frac{1}{2} \int V\left(\boldsymbol{k}_{1}, \boldsymbol{k}_{2}, \boldsymbol{k}_{3}\right) \\
& a_{\boldsymbol{k}_{2}} a_{\boldsymbol{k}_{3}} \delta\left(\boldsymbol{k}_{1}-\boldsymbol{k}_{2}-\boldsymbol{k}_{3}\right) \mathrm{d} \boldsymbol{k}_{2} \mathrm{~d} \boldsymbol{k}_{3}+\ldots,
\end{aligned}
$$

so that the time derivative of a wave amplitude (with some wave vector $\boldsymbol{k}_{1}$ ) is determined by the contributions of all other waves. The dispersion law $\omega(\boldsymbol{k})$ determines the linear part of Eq. (6), and the coupling coefficient $V\left(\boldsymbol{k}_{1}, \boldsymbol{k}_{2}, \boldsymbol{k}_{3}\right)$ determines quadratic nonlinearity. The dots stand for other nonlinear terms; these are other quadratic terms (namely, the ones containing $a a^{*}$ and $a^{*} a^{*} ;{ }^{*}$ denotes complex conjugation), as well as higher order nonlinearity terms. The system is obviously translationally symmetric with respect to shifts in time (since all coefficients are time-independent), so it conserves the energy. The system is also translationally symmetric with respect to shifts in space, due to the presence of the delta function of the wave vectors (it just provides a way to write the convolution), so the system conserves momentum.

Zakharov and Schulman assumed that the wave amplitudes are chosen to be canonical Hamiltonian variables, so that the dynamical Eq. (6) has the form

$i \frac{\partial}{\partial t} a_{k}=\frac{\delta \mathcal{H}}{\delta a_{k}^{*}}$

with some Hamiltonian $\mathcal{H}$ expanded in a series over powers of wave amplitudes

$$
\begin{aligned}
\mathcal{H} & =\int \omega(\boldsymbol{k}) a_{\boldsymbol{k}} a_{\boldsymbol{k}}^{*} \mathrm{~d} \boldsymbol{k} \\
& +\frac{1}{2} \int V\left(\boldsymbol{k}_{1}, \boldsymbol{k}_{2}, \boldsymbol{k}_{3}\right) a_{\boldsymbol{k}_{1}}^{*} a_{\boldsymbol{k}_{2}} a_{\boldsymbol{k}_{3}} \delta\left(\boldsymbol{k}_{1}-\boldsymbol{k}_{2}-\boldsymbol{k}_{3}\right) \mathrm{d} \boldsymbol{k}_{2} \mathrm{~d} \boldsymbol{k}_{3} \\
& +\{\text { c.c. }\}+\{\ldots \text { other nonlinear terms ... }\}
\end{aligned}
$$

( $\{c . c$.$\} means the complex conjugated term). Then the mo-$ mentum is

$\boldsymbol{P}=\int \boldsymbol{k} a_{\boldsymbol{k}} a_{\boldsymbol{k}}^{*} \mathrm{~d} \boldsymbol{k}$

and the energy is the Hamiltonian. Zakharov and Schulman investigated when the wave system has another conservation 
law:

$$
\begin{aligned}
I & =\int \varphi(\boldsymbol{k}) a_{\boldsymbol{k}} a_{\boldsymbol{k}}^{*} \mathrm{~d} \boldsymbol{k} \\
& +\frac{1}{2} \int F\left(\boldsymbol{k}_{1}, \boldsymbol{k}_{2}, \boldsymbol{k}_{3}\right) a_{\boldsymbol{k}_{1}}^{*} a_{\boldsymbol{k}_{2}} a_{\boldsymbol{k}_{3}} \delta\left(\boldsymbol{k}_{1}-\boldsymbol{k}_{2}+\boldsymbol{k}_{3}\right) \mathrm{d} \boldsymbol{k}_{2} \mathrm{~d} \boldsymbol{k}_{3} \\
& +\{c . c .\}+\{\ldots \text { other nonlinear terms } \ldots\}
\end{aligned}
$$

(with undetermined $\varphi, F$, and so on); $\varphi$ is supposed to be linearly independent of $\boldsymbol{k}$ and $\omega$, so that $I$ is independent of the momentum and energy. They found that in order for Eq. (10) to be conserved by the dynamics in Eq. (7)-(8), the following alternative should hold. At least one of the two conditions are to be satisfied:

1. The function $\varphi(\boldsymbol{k})$ is conserved in the resonant triad interactions: the equation

$\varphi\left(\boldsymbol{k}_{1}\right)=\varphi\left(\boldsymbol{k}_{2}\right)+\varphi\left(\boldsymbol{k}_{3}\right)$

holds for all vectors $\boldsymbol{k}_{1}, \boldsymbol{k}_{2}$, and $\boldsymbol{k}_{3}$ bound by the resonance relations

$$
\boldsymbol{k}_{1}=\boldsymbol{k}_{2}+\boldsymbol{k}_{3}, \quad \omega\left(\boldsymbol{k}_{1}\right)=\omega\left(\boldsymbol{k}_{2}\right)+\omega\left(\boldsymbol{k}_{3}\right) .
$$

2. Or the resonant triad interaction is impossible. This can happen in two ways: Eq. (12) can have no solutions (like it happens for the gravity waves); or the nonlinear coupling coefficient $V\left(\boldsymbol{k}_{1}, \boldsymbol{k}_{2}, \boldsymbol{k}_{3}\right)$ can identically vanish on the resonance manifold defined by Eqs. (12).

Introducing the classical scattering matrix, Zakharov and Schulman (1988) obtained other necessary conditions for the conservation of Eq. (10). These are equations on $F\left(\boldsymbol{k}_{1}, \boldsymbol{k}_{2}, \boldsymbol{k}_{3}\right)$ and other kernels of higher order nonlinear terms in Eq. (10). They demonstrated $(1980 ; 1988)$ their arguments on several integrable systems, in particular, the Kadomtsev-Petviashvili equation.

They also called the dispersion law $\omega(\boldsymbol{k})$ - which admits the additional conservation in Eq. (11) - degenerative.

The Zakharov-Schulman Eq. (11) and (12) for nonlinear waves are similar to Boltzmann's Eqs. (4) and (1) for rarefied gas, but waves provide a bigger variety:

a. The main interactions can involve any number of waves, in particular, only three waves (like in Eqs. 1112).

b. There are many forms of the dispersion law, not just the quadratic function $\omega(\boldsymbol{k})=\boldsymbol{k}^{2}$ (like in Boltzmann's case).

c. The physically interesting media (in which the waves propagate) can have different dimensions of $d=$ $1,2,3$.

\section{Unique conservation properties of large-scale geophysical fluid dynamics}

\subsection{Quasi-geostrophic equation}

The Rossby wave dispersion law turned out to be degenerative (Balk et al., 1991; Balk, 1991).

When dispersion law has the form

$\omega(\boldsymbol{k})=-\frac{\beta p}{\alpha^{2}+k^{2}} \quad\left[\boldsymbol{k}=(p, q), \quad k^{2}=p^{2}+q^{2}\right]$

( $\alpha$ and $\beta$ are parameters), the resonance relations in Eq. (12) imply Eq. (11) with $\varphi(\boldsymbol{k})$ equal to the function

$\eta(\boldsymbol{k}) \equiv \arctan \frac{\alpha(q+p \sqrt{3})}{k^{2}}-\arctan \frac{\alpha(q-p \sqrt{3})}{k^{2}}$

(Balk, 1991). The conservation of the function in Eq. (14) in triad resonances implies that the quasi-geostrophic equation

$\left(\Delta \psi-\alpha^{2} \psi\right)_{t}+\beta \psi_{x}+\psi_{y} \Delta \psi_{x}-\psi_{x} \Delta \psi_{y}=0$

possesses an approximate invariant

$I=\frac{1}{2} \int \frac{1}{p} \eta(\boldsymbol{k})\left|\mathcal{Q}_{\boldsymbol{k}}(t)\right|^{2} \mathrm{~d} p \mathrm{~d} q$,

with $\mathcal{Q}_{\boldsymbol{k}}(t)$ being the Fourier transform of

$\mathcal{Q}(x, y, t)=\Delta \psi-\alpha^{2} \psi$.

The function in Eq. (14) is conserved exactly in resonant triad interactions, but the integral in Eq. (16) is conserved approximately in all interactions (including triads that are far from being resonant). (If typical length scale $L$ and parameter $\beta$ are normalized to 1 , and $\varepsilon$ is a characteristic nondimensional wave amplitude, then the invariant in Eq. (16) has a magnitude of $O\left(\varepsilon^{2}\right)$, while its variation $\Delta I \equiv I(t)-$ $I(0)$ has a smaller magnitude of $\Delta I=O\left(\varepsilon^{3}\right)$ on long time intervals $O(1 / \varepsilon)$; see Balk and van Heerden, 2006, for details.)

Ferapontov (1992) realized that the problem of collision invariants (for rarefied gas, Sect. 1.1) and the problem of degenerative dispersion laws (for nonlinear waves, Sect. 1.2) can be posed as a problem of web geometry, which has been developing since the 1920s (Blaschke, 1932).

In particular, using the web geometry results, it is possible to show (Balk and Ferapontov, 1998) that the function in Eq. (14) is unique: any function $\varphi(\boldsymbol{k})$, conserved in triad resonances in Eq. (12) - with the Rossby dispersion law $\omega(\boldsymbol{k})-$ is a linear combination of $\boldsymbol{k}, \omega(\boldsymbol{k})$, and $\eta(\boldsymbol{k})$.

The connection to the web geometry also shows how exceptional the systems with extra invariants are. As one can guess, it is rare that four Eqs. (11) and (12) (the first equation in (12) consists of two scalar equations) in the sixdimensional space of wave vectors $\boldsymbol{k}_{1}, \boldsymbol{k}_{2}, \boldsymbol{k}_{3}$ determine a manifold of dimension 3. And it is indeed extremely rare (see Balk and Ferapontov, 1998, for details). 
Even though Eq. (11) follows from the resonance relations in Eq. (12) (Eq. 11 is only linearly independent of Eq. 12), the integral (Eq. 16) is completely independent of the standard invariants (energy-momentum), because the integrand contains $\mathcal{Q}_{\boldsymbol{k}}(t)$.

The presence of the extra invariant in Eq. (16) implies the energy transfer from small-scale eddies to large-scale zonal jets (Balk, 2005).

This is often but not always the case: the extra invariant does not lead to zonal jets, when the energy source is on large scales (i.e., the forcing scale $k_{\mathrm{f}}$ is long-wave, $k_{\mathrm{f}} \ll \alpha$ ). In the latter situation, the energy accumulates in the sectors of polar angles $\theta=\arctan (q / p)$ such that $60^{\circ}<|\theta|<90^{\circ}$. However, in the present paper we do not need to deal with this, since we are concerned with the opposite situation $k \gg \alpha$ for all $k$. As $\boldsymbol{k} \rightarrow \infty$, the extra invariant function in Eq. (14) and the dispersion law in Eq. (13) turn out to be asymptotically proportional (the same proportionality in all directions $\boldsymbol{k} / \boldsymbol{k}$ !); a certain linear combination of Eqs. (14) and (13), which cancels the common asymptotics, gives an extra invariant

$$
\frac{5}{4 \alpha^{4}}\left[\frac{\eta(\boldsymbol{k})}{2 \sqrt{3} \alpha}+\frac{\omega(\boldsymbol{k})}{\beta}\right] \sim \frac{p^{3}\left(p^{2}+5 q^{2}\right)}{k^{10}}, \quad(k \gg \alpha) .
$$

This linear combination decreases much faster with $|\boldsymbol{k}|$ than both functions in Eq. (14) and (13); the energy transfer towards zonal jets turns out to be more pronounced in the shortwave case (see Balk, 2005, for details of long- and shortwave limits).

Nazarenko and Quinn (2009) demonstrated the conservation of the extra invariant in Eq. (18) in the numerical simulations of the quasi-geostrophic equation.

\subsection{Shallow water system}

The extra invariant in Eq. (16) was extended (Balk et al., 2011) to the shallow water system on the beta plane

$$
\begin{aligned}
& u_{t}+u u_{x}+v u_{y}-f(y) v=-g H_{x}, \\
& v_{t}+u v_{x}+v v_{y}+f(y) u=-g H_{y}, \\
& H_{t}+(H u)_{x}+(H v)_{y}=0,
\end{aligned}
$$

where $(u, v)$ is the horizontal fluid velocity, $H$ the fluid height (flat bottom is assumed), $g$ the gravity acceleration, and $f(y)$ the Coriolis parameter. The shallow water system in Eq. (19) adiabatically conserves the integral in Eq. (16), with $\mathcal{Q}_{k}(t)$ being the Fourier transform of the perturbational potential vorticity (e.g., Gill, 1982)

$\mathcal{Q}(x, y, t)=v_{x}-u_{y}-f(y) h$,

where $h=\left(H-H_{0}\right) / H_{0}$ is the fractional relative height, which measures the deviation of the fluid surface height $H(x, y, t)$ from its unperturbed value $H_{0}$.

The extension was possible due to the following two unique conservation properties of Eq. (19).
First, the shallow water dynamics in Eq. (19) - besides Rossby waves - contains the inertia-gravity (IG) waves. The former could (in general) leak energy to the latter, thereby destroying the conservation of the extra invariant in Eq. (16) which is based only on Rossby waves. However, the coupling coefficient of triad interaction between the IG and the Rossby waves vanishes, provided the Coriolis parameter is constant. If $f(y)$ varies sufficiently slowly (i.e., $\beta(y) \equiv f^{\prime}(y)$ is sufficiently small), then the extra invariant slowly changes due to $\beta \neq 0$, but within its non-conservation due to the quartet interactions (recall that the invariant is only adiabatically conserved and can slowly change).

Second, the beta plane in Eq. (19) can be not only midlatitudinal but also close to the Equator. As we approach the Equator, the Coriolis parameter decreases, and the quasigeostrophic approximation becomes invalid. Correspondingly, in the perturbation expansions, the Coriolis parameter $f$ enters the denominators. However, the specific form of Eq. (19) makes it possible to refine (Balk et al., 2011) the Rossby mode, so that the numerators (in the perturbation expansion) are equally small in the limit $f \rightarrow 0$.

\section{$2.3 \quad 3-D$ fluid layers}

\subsubsection{Motivation}

The application of the extra invariant in Eq. (16) to the real oceans and atmospheres of rotating planets encounters the following natural question. In a fluid layer of finite depth $H$, the shallow water motions (which are two-dimensional, 2-D) can generate - due to nonlinearity - the 3-D inertia waves. One can argue that the length of a typical Rossby wave significantly exceeds the depth $H$, so the energy exchange between Rossby waves and inertia waves can be disregarded due to the disparity of scales. But the question remains for short Rossby waves, whose wavelength can be comparable to the depth $H$; they could effectively interact with 3-D modes. Even if the energy of short Rossby waves were small, they could gradually leak significant amount of energy during long time evolution.

At the same time, the extra conservation seems to be essentially tied to the two-dimensionality or, more generally, to low dimension. So far, extra invariants have been found in two situations: (1) triads in 2-D media (see Eq. 12) and (2) quartets in 1-D media (Balk and Ferapontov, 1993), e.g.,

$p_{1}+p_{2}=p_{3}+p_{4}, \omega\left(p_{1}\right)+\omega\left(p_{2}\right)=\omega\left(p_{3}\right)+\omega\left(p_{4}\right)$

( $p$ is the wave number in the 1-D media). In both situations, a wave with some fixed wave vector (say, $\boldsymbol{k}_{1}$ or $p_{1}$ ) participates in only one-dimensional family of resonant interactions. Let us elaborate. The triad resonance relations in Eq. (12) for 2 $\mathrm{D}$ wave vectors define manifold of dimension 3 . A wave with a fixed wave vector (say, $\boldsymbol{k}_{1}$ ) can be involved with only onedimensional family of resonant triads. The quartet resonance manifold in Eq. (21) has a dimension of two; a fixed wave 
vector (say $p_{1}$ ) participates again in one-dimensional family of resonant interactions.

For 3-D media, the triad resonance relations in Eq. (12) define manifold of dimension 5; a fixed wave vector (say, $\boldsymbol{k}_{1}$ ) is involved in two-dimensional family of resonance triads. There is an example of extra invariants for triad interaction in 3-D media (Balk, 1997), but it seems somewhat artificial.

Nevertheless, the extra invariant in Eq. (16) can be extended to the 3-D dynamics. This is due to the special property of the fluid equations, which was found a long time ago (Greenspan, 1969).

\subsubsection{Problem}

Since the short Rossby waves are the prime candidates for the energy exchange with 3-D modes, we consider the 3-D dynamics in the well-known classical situation (e.g., Landau and Lifshitz, 1987) of ideal incompressible (with constant density $\rho_{0}$ ) rotating fluid

$$
\begin{aligned}
\boldsymbol{v}_{t}+(\boldsymbol{v} \cdot \nabla) \boldsymbol{v}+\boldsymbol{f} \times \boldsymbol{v} & =-\nabla \Pi, \\
\nabla \cdot \boldsymbol{v} & =0,
\end{aligned}
$$

where $\boldsymbol{v}=(u, v, w)$ is the fluid velocity; $\Pi$ is the fluid pressure (centrifugal force included) divided by $\rho_{0}$. Euler's equation (Eq. 22) involves Coriolis force, with $\boldsymbol{f}$ being the double angular velocity. The fluid is assumed to occupy a layer between two parallel horizontal planes, rotating about vertical axis $z$. The boundary condition is vanishing of the vertical fluid velocity at the solid plane boundaries

$w(x, y, 0, t)=w\left(x, y, H_{0}, t\right)=0$.

The Coriolis parameter slowly changes with the meridional coordinate $y$ :

$\boldsymbol{f}=(0,0, f), \quad f=f_{0}+\beta y$.

The system in Eq. (22) describes the dynamics of inertia waves and vortical mode (e.g., Landau and Lifshitz, 1987, $\S 14)$; the latter - due to the dependence of $f$ on $y$ - represents short Rossby waves with dispersion law

$\Omega(\boldsymbol{k})=-\beta \frac{p}{k^{2}} \quad[\boldsymbol{k}=(p, q)]$.

Greenspan (1969) discovered that on the $f$ plane (when $\beta=0$ ), the inertia modes do not produce triad-resonant response on the geostrophic mode. In other words, in the equation for the geostrophic mode (which has zero frequency), the coupling coefficient between this mode and two inertia modes (with frequencies $\omega_{m}$ and $\omega_{n}$ ) vanishes whenever $\omega_{m}+\omega_{n}=0$. It is reasonable to think that for nonzero - but sufficiently small $-\beta$, the triad resonant response is sufficiently small, so the second condition of ZakharovSchulman's alternative (Sect. 1.2) is realized approximately: $V\left(\boldsymbol{k}_{1}, \boldsymbol{k}_{2}, \boldsymbol{k}_{3}\right)$ on the triad resonance manifold is not zero but "small". Then, the rapidly rotating 3-D fluid dynamics appears to have the invariants of the 2-D shallow water dynamics. In Sect. 3 this is derived explicitly.

\subsubsection{Result}

Let $L$ be the length scale, $U_{0}$ the velocity scale, and $f_{0}=$ $f(0)$ the reference value of the Coriolis parameter. We use two small parameters:

- First, the Coriolis parameter $f(y)$ is a slow function: the variation $\Delta f \equiv f-f_{0} \sim \beta L$ is small compared to $f_{0}$

$\frac{\beta L}{f_{0}} \ll 1$.

This condition means that $L$ is much less than the Earth radius $R_{0}$, since at midlatitudes, $\beta \sim f_{0} / R_{0}$.

- Second, we consider weakly nonlinear dynamics, when the nonlinear terms are small compared to the linear terms. This means nonlinearity being small compared to the beta effect, namely,

$\epsilon=\frac{U_{0}}{\beta L^{2}} \ll 1$.

We need this, even though the Euler equation (Eq. 22) contains a linear term with $f$. Indeed, later (Sect. 3.2), we consider Eq. (33) for the vertical component of vorticity. We integrate Eq. (33) over the fluid depth, and the $f$ term disappears (due to the incompressibility $u_{x}+v_{y}+w_{z}=0$ and boundary condition in Eq. (23)). Then the only remaining linear term contains $\beta$, and the typical ratio of nonlinear terms to linear term is $\epsilon$.

Let us estimate $\epsilon$ for large-scale dynamics in Earth's oceans: flow at latitude $\approx 30^{\circ}$ with $U_{0} \approx 5 \mathrm{~cm} \mathrm{~s}^{-1}$ and $L \approx 100 \mathrm{~km}$ has $\epsilon \approx 1 / 4$. Is this sufficiently small to have the extra invariant? Perhaps, the weakly nonlinear dynamics is just a useful model that cannot be fully justified but nevertheless leads to plausible results (see also discussion of weak nonlinearity in the Introduction).

At this point, we assume that the fluid equations are written in dimensionless form with timescale $1 / f_{0}$ and length scale $L$ (in other words, the units are chosen such that $f_{0}=L=1$ ). Then the dimensionless Coriolis parameter is $f=1+\beta y$ with dimensionless $\beta \ll 1$; the dimensionless typical fluid velocity is $U_{0}=\epsilon \beta$.

We will derive in Sect. 3 that the dynamics in Eq. (22)(24) possesses an invariant

$I=\frac{1}{2} \int \frac{p^{2}\left(p^{2}+5 q^{2}\right)}{\left(p^{2}+q^{2}\right)^{5}}\left|\zeta_{\boldsymbol{k}}(t)\right|^{2} \mathrm{~d} \boldsymbol{k}$,

where $\boldsymbol{k}=(p, q)$ is the horizontal wave vector, and $\zeta_{\boldsymbol{k}}(t)$ is the Fourier transform of $z$-averaged vertical vorticity

$\zeta(x, y, t)=\frac{1}{H_{0}} \int_{0}^{H_{0}}\left(v_{x}-u_{y}\right) \mathrm{d} z$. 
We will derive the following estimate for the variation of the integral in Eq. (25):

$\Delta I \equiv I(t)-I(0)=O\left(\epsilon^{2.5} \beta^{2}\right)+O\left(\epsilon^{2} \beta^{2.5}\right)$,

and over time $t=O\left(\epsilon^{-1} \beta^{-1}\right)$. At the same time, the invariant $I$ has the order $\epsilon^{2} \beta^{2}$, so $\Delta I \ll I$. The period of Rossby waves is $O\left(\beta^{-1}\right)$, so $I$ is conserved during time intervals of many - namely $O\left(\epsilon^{-1}\right)$ - Rossby wave periods. (An estimate stronger than Eq. (27) can be obtained with further assumptions.)

\section{Derivation}

\subsection{Normal mode expansion}

To obtain the extra invariant in Eq. (25), let us expand the solution of Eq. (22) in normal modes of linearized system with $\beta=0$ :

$$
\begin{aligned}
u_{t}-f_{0} v & =-\Pi_{x}, \\
v_{t}+f_{0} u & =-\Pi_{y}, \\
w_{t} & =-\Pi_{z}, \\
u_{x}+v_{y}+w_{z} & =0, \\
w(x, y, 0, t)=w\left(x, y, H_{0}, t\right) & =0 .
\end{aligned}
$$

(In our dimensionless units $f_{0}=1$, but we keep $f_{0}$ just to see physical meaning.) There are normal modes of two types: (1) the vortical mode, representing the Taylor-Proudman column

$u=-\frac{1}{f_{0}} \Pi_{y}, \quad v=\frac{1}{f_{0}} \Pi_{y}, \quad w=0, \quad \Pi_{z}=0$,

(which is $t$ and $z$ independent), and (2) the inertial waves (standing in the $z$ direction)

$$
\left[\begin{array}{c}
u \\
v \\
w \\
\Pi
\end{array}\right]=\left[\begin{array}{c}
\hat{u} \cos m z \\
\hat{v} \cos m z \\
\hat{w} \sin m z \\
\hat{\Pi} \cos m z
\end{array}\right] e^{i(p x+q y-\omega t)},
$$

where $m=n \pi / H_{0}$ with non-zero integer $n$. Substitution of Eq. (30) into Eq. (28) gives the dispersion relation

$\omega^{2} K^{2}=f_{0}^{2} m^{2} \quad\left(K^{2}=p^{2}+q^{2}+m^{2}\right)$,

and the polarization vector

$$
\left[\begin{array}{c}
\hat{u} \\
\hat{v} \\
\hat{w} \\
\hat{\Pi}
\end{array}\right]=\left[\begin{array}{c}
-i q f_{0}-\omega p \\
i p f_{0}-\omega q \\
i \omega k^{2} / m \\
f_{0}^{2}-\omega^{2}
\end{array}\right] .
$$

The capital $\boldsymbol{K}$ denotes the full three-dimensional wave vector, $\boldsymbol{K}=(p, q, m)$, while the small case $\boldsymbol{k}$ stands for the horizontal 2-D vector, $\boldsymbol{k}=(p, q)$. The general solution of Eq. (28) is a linear combination of all normal modes with some coefficients $\Psi_{\boldsymbol{k}}$ and $A_{\boldsymbol{K}}$ :

$$
\begin{gathered}
{\left[\begin{array}{c}
u(x, y, z, t) \\
v(x, y, z, t) \\
w(x, y, z, t) \\
\Pi(x, y, z, t)
\end{array}\right]=\int \mathrm{d} p \mathrm{~d} q e^{i(p x+q y)}} \\
\left\{\Psi_{\boldsymbol{k}}\left[\begin{array}{r}
-i q \\
i p \\
0 \\
f_{0}
\end{array}\right]+\sum_{m} A_{\boldsymbol{K}}\left[\begin{array}{c}
\left(-i q f_{0}-\omega p\right) \cos m z \\
\left(i p f_{0}-\omega q\right) \cos m z \\
\left(i \omega k^{2} / m\right) \sin m z \\
\left(f_{0}^{2}-\omega^{2}\right) \cos m z
\end{array}\right] e^{-i \omega t}\right\} .
\end{gathered}
$$

Here $\omega \equiv f_{0} m / \boldsymbol{K}$; the summation in Eq. (32) is over $m=$ $\frac{\pi}{H_{0}} n, n= \pm 1, \pm 2, \ldots ; \Psi_{-k}=\Psi_{k}^{*}, A_{-K}=A_{K}^{*}$.

The solution of the full (nonlinear and with non-zero $\beta$ ) system in Eq. (22) can also be expanded in the form (32), but the coefficients $\Psi_{\boldsymbol{k}}$ and $A_{\boldsymbol{K}}$ would depend on time $t$.

\subsection{Equation for the vertical vorticity in terms of normal modes}

From Eq. (22) we have

$$
\begin{aligned}
\left(v_{x}-u_{y}\right)_{t} & +\left(u v_{x}+v v_{y}+w v_{z}\right)_{x} \\
& -\left(u u_{x}+v u_{y}+w u_{z}\right)_{y}+f\left(u_{x}+v_{y}\right)+\beta v=0 .
\end{aligned}
$$

Now we substitute Eq. (32) into Eq. (33) and integrate over $z$ (from 0 to $H_{0}$ ), taking into account that cosines $\cos m z$ integrate to zero. To shorten writing, any subscript wave vector $\boldsymbol{k}_{j}$ or $\boldsymbol{K}_{j}$ will be replaced by its label subindex $j$ (e.g., $\Psi_{j} \equiv \Psi_{\boldsymbol{k}_{j}}, A_{j} \equiv A_{\boldsymbol{K}_{j}}$ ). This is unambiguous: index $j$ stands for $\boldsymbol{K}_{j}$ if the corresponding quantity - like $A$ - depends on the three-dimensional wave vector $\boldsymbol{K} ; j$ stands for $\boldsymbol{k}_{j}$ if the corresponding quantity - like $\Psi$ - depends on the twodimensional wave vector $\boldsymbol{k}$. In addition, $-j$ stands for $-\boldsymbol{K}_{j}$ or $-\boldsymbol{k}_{j} ; \mathrm{d}_{23} \equiv \mathrm{d} \boldsymbol{k}_{2} \mathrm{~d} \boldsymbol{k}_{3} \equiv \mathrm{d} p_{2} \mathrm{~d} q_{2} \mathrm{~d} p_{3} \mathrm{~d} q_{3}$.

Thus, Eq. (33) becomes

$$
\begin{aligned}
k_{1}^{2} \dot{\Psi}_{1} & =\beta i p_{1} \Psi_{1}+\frac{1}{2} \int W_{-1,2,3} \Psi_{2} \Psi_{3} \mathrm{~d}_{23} \\
& +\frac{1}{2} \int \sum_{m_{2}, m_{3}} V_{-1,2,3} e^{-i\left(\omega_{2}+\omega_{3}\right) t} A_{2} A_{3} \mathrm{~d}_{23} .
\end{aligned}
$$

The integrals in Eq. (34) have been symmetrized with respect to transposition of indexes 2 and 3 : this gives the factor $1 / 2$ in front of the integrals and the symmetric coupling coefficients $\left(W_{-1,2,3}=W_{-1,3,2}\right.$ and $\left.V_{-1,2,3}=V_{-1,3,2}\right)$

$$
\begin{aligned}
W_{1,2,3} \equiv & W_{\boldsymbol{k}_{1}, \boldsymbol{k}_{2}, \boldsymbol{k}_{3}} \\
& =\Delta_{1,2,3}\left(k_{3}^{2}-k_{2}^{2}\right) \delta\left(\boldsymbol{k}_{1}+\boldsymbol{k}_{2}+\boldsymbol{k}_{3}\right), \\
V_{1,2,3} & \equiv V_{\boldsymbol{k}_{1}, \boldsymbol{K}_{2}, \boldsymbol{K}_{3}} \\
& =\left[\left(f_{0} \Delta_{1,2,3}-i \omega_{3} \boldsymbol{k}_{1} \cdot \boldsymbol{k}_{\mathbf{3}}\right)\left(f_{0} \boldsymbol{k}_{1} \cdot \boldsymbol{k}_{\mathbf{2}}-i \omega_{2} \Delta_{123}\right)\right. \\
& \left.-\left(f_{0} \Delta_{1,2,3}+i \omega_{2} \boldsymbol{k}_{1} \cdot \boldsymbol{k}_{\mathbf{2}}\right)\left(f_{0} \boldsymbol{k}_{1} \cdot \boldsymbol{k}_{\mathbf{3}}+i \omega_{3} \Delta_{123}\right)\right] \\
& \left(\delta_{m_{2}-m_{3}}+\delta_{m_{2}+m_{3}}\right) \delta\left(\boldsymbol{k}_{1}+\boldsymbol{k}_{2}+\boldsymbol{k}_{3}\right) .
\end{aligned}
$$


Here $\delta(\cdot)$ is the Dirac delta function, and $\delta_{m}$ is the Kronecker symbol (which is 1 if $m=0$ and 0 if $m \neq 0$ ):

$\Delta_{1,2,3}=p_{2} q_{3}-p_{3} q_{2}$,

which - because of the delta function - has the following obvious symmetries:

$\Delta_{1,2,3}=\Delta_{2,3,1}=\Delta_{3,1,2}=-\Delta_{1,3,2}=-\Delta_{3,2,1}=-\Delta_{2,1,3}$.

That is, $\Delta_{1,2,3}$ is symmetric with respect to the three cyclic permutations of its indexes and anti-symmetric with respect to the other three permutations. Software Mathematica has been used to obtain $V$ in the form of Eq. (36).

\subsection{Adiabatic conservation}

We intend to find invariant

$I=\frac{1}{2} \int X(k) \Psi_{k} \Psi_{-k} \mathrm{~d} \boldsymbol{k}, \quad[X(k)=X(-k)]$

(with undetermined kernel $X$ ), which are approximately conserved over a long time $O\left(\epsilon^{-1} \beta^{-1}\right)$. This is the nonlinear time of wave interactions (since the wave amplitudes are of the order of fluid velocity, which is $O(\epsilon \beta)$ ). To show that $I(t)$ stays almost constant, it would be enough to show that its time derivative $\dot{I}$ is sufficiently small. However, the latter is not the case: $I(t)$ oscillates in time (similar to adiabatic invariants in the theory of dynamical systems). The change $\Delta I \equiv I(t)-I(0)$ is "small", but $\dot{I}$ is "big". The usual way to deal with such a situation has been the following. The quadratic integral in Eq. (37) is supplemented by cubic corrections, e.g., for the dynamics in Eq. (34):

$$
\begin{aligned}
I^{\text {suppl. }}= & \frac{1}{2} \int X(\boldsymbol{k}) \Psi_{\boldsymbol{k}} \Psi_{-\boldsymbol{k}} \mathrm{d} \boldsymbol{k} \\
& +\int Y_{123} \Psi_{1} \Psi_{2} \Psi_{3} \mathrm{~d}_{123}+\int Z_{123} \Psi_{1} A_{2} A_{3} \mathrm{~d}_{123}
\end{aligned}
$$

( $X, Y, Z$ are undetermined kernels); $\dot{I}^{\text {suppl. }}$ is required to be sufficiently small. At the end, the cubic corrections can be dropped, since they turn out to be within the variation of $\Delta I^{\text {suppl. }} \equiv I^{\text {suppl. }}(t)-I^{\text {suppl. }}(0)$.

Here we develop a new, more direct, approach (without cubic corrections).

Let us, first, apply the transformation

$\Psi_{k}=\psi_{k} e^{-i \Omega(k) t}$;

it removes the linear term in Eq. (34),

$$
\begin{aligned}
\dot{\psi}_{1}= & \frac{1}{2 k_{1}^{2}} \int W_{-1,2,3} e^{i\left(\Omega_{1}-\Omega_{2}-\Omega_{3}\right) t} \psi_{2} \psi_{3} \mathrm{~d}_{23} \\
& +\frac{1}{2 k_{1}^{2}} \int \sum_{m_{2}, m_{3}} V_{-1,2,3} e^{i\left(\Omega_{1}-\omega_{2}-\omega_{3}\right) t} A_{2} A_{3} \mathrm{~d}_{23},
\end{aligned}
$$

and leaves the invariant (37) in the old form

$I=\frac{1}{2} \int X(\boldsymbol{k}) \psi_{\boldsymbol{k}} \psi_{-\boldsymbol{k}} \mathrm{d} \boldsymbol{k}$.

Its time derivative is

$\dot{I}=\dot{I}^{\mathrm{R}}+\dot{I}^{\mathrm{i}}$,

where

$$
\begin{aligned}
\dot{I}^{\mathrm{R}} & =\frac{1}{2} \int \mathrm{d}_{123} \frac{1}{k_{1}^{2}} W_{123} e^{-i\left(\Omega_{1}+\Omega_{2}+\Omega_{3}\right) t} \psi_{1} \psi_{2} \psi_{3}, \\
\dot{I}^{\mathrm{i}} & =\frac{1}{2} \int \mathrm{d}_{123} \sum_{m_{2}, m_{3}} X_{1} \frac{1}{k_{1}^{2}} V_{123} \psi_{1} e^{-i\left(\Omega_{1}+\omega_{2}+\omega_{3}\right) t} A_{2} A_{3} ;
\end{aligned}
$$

$\dot{I}^{\mathrm{R}}$ and $\dot{I}^{\mathrm{i}}$ are respectively contributions to $\dot{I}$ resulting from the interaction between three Rossby waves and from the interaction between two inertia waves and one Rossby wave. In both integrals in Eq. (41), we have replaced $\boldsymbol{k}_{1}$ by $-\boldsymbol{k}_{1}$ and used that the dispersion law $\Omega$ is an odd function $\left(\Omega_{-1}=\right.$ $-\Omega_{1}$ ). Our goal is to estimate the contributions to $\Delta I$ resulting from $\dot{I}^{\mathrm{R}}$ and $\dot{I}^{\mathrm{i}}$, and thereby show that $\Delta I \ll I$.

\subsection{Small denominator lemma}

The essence of the extra invariant conservation can be expressed by the lemma below. Basically, we have

$$
\frac{\mathrm{d} I}{\mathrm{~d} \tau}=\int_{-\infty}^{\infty} F(\chi, \varepsilon \tau) e^{i \chi \tau} \mathrm{d} \chi,
$$

and need to estimate the total variation $\Delta I \equiv I(\tau)-I(0)$ over a long time interval $0 \leq \tau \leq \varepsilon^{-1}$.

For instance, in the case of Eq. (41), the variable $\chi$ results from the exponent, and the function $F$ is the result of all integrations while $\chi$ is held fixed (later, when applying the lemma, we will define $\chi$ and $F$ exactly). The function $F$ slowly depends on time $\tau$, which is signified by a small parameter $\varepsilon$ (this corresponds to the slow variation of wave amplitudes). The integral in Eq. (42) is well convergent at $\chi \rightarrow \pm \infty$ (corresponding to the vanishing of wave amplitudes as $\boldsymbol{k}_{1}, \boldsymbol{k}_{2}$, and $\boldsymbol{k}_{3}$ approach infinity). It is important that the integration interval in Eq. (42) includes point $\chi=0$, so that the resonance (at $\chi \rightarrow 0$ ) is present.

In general, $I$ exhibits secular growth associated with the resonance $\chi \rightarrow 0$, and the adiabatic conservation of $I$ is out of the question. Indeed, assume for simplicity that $F$ is time independent $(\varepsilon=0)$; then

$$
\Delta I=\int F(\chi) \frac{e^{i \chi \tau}-1}{i \chi} \mathrm{d} \chi
$$

here $i \chi$ is the "small denominator" ("small divisor"), corresponding to the resonance $\chi \rightarrow 0$. According to Eq. (43), for large $\tau$, the increment $\Delta I$ grows proportional to $\tau$, unless the small denominator is somehow cancelled. 
For LEMMA,

Suppose, there are constants $M$ and $L$ such that

$$
\begin{aligned}
& \int_{-\infty}^{\infty}\left|\frac{F(\chi, \mathcal{T})}{\chi}\right| \mathrm{d} \chi \leq M, \\
& \int_{-\infty}^{\infty}\left|F\left(\chi, \mathcal{T}^{\prime \prime}\right)-F\left(\chi, \mathcal{T}^{\prime}\right)\right| \mathrm{d} \chi \leq L\left|\mathcal{T}^{\prime \prime}-\mathcal{T}^{\prime}\right|
\end{aligned}
$$

for all positive $\mathcal{T}, \mathcal{T}^{\prime}, \mathcal{T}^{\prime \prime}$. Then

$$
|\Delta I| \leq(2 M+L) \varepsilon^{-1 / 2} \text { for } 0 \leq \tau \leq \varepsilon^{-1} .
$$

Comment 1. The convergence of integral in Eq. (44) implies cancellation (at least partial) of the small denominator $\chi$.

Comment 2. The constants $M$ and $L$ are $\mathcal{T}$-independent, but can depend on $\varepsilon$, which will be used in the lemma applications below.

Proof. We split the time interval $0 \leq \tau \leq \varepsilon^{-1}$ into many subintervals

$$
\left[\tau_{j-1}, \tau_{j}\right], \quad j=1,2, \ldots J \quad\left(\tau_{0}=0, \quad \tau_{J}=\varepsilon^{-1}\right),
$$

of length $\varepsilon^{-1 / 2}$; there are $J=\varepsilon^{-1 / 2}$ of them. (To make this number integer, we can consider an appropriate subsequence of $\varepsilon$, or we can use the integer part $J=\left[\varepsilon^{-1 / 2}\right]+1$ with slightly shorter subintervals.) On each subinterval

$$
\frac{\mathrm{d} I}{\mathrm{~d} \tau}=\int\left\{F\left(\chi, \varepsilon \tau_{j}\right)+\left[F(\chi, \varepsilon \tau)-F\left(\chi, \varepsilon \tau_{j}\right)\right]\right\} e^{i \chi \tau} \mathrm{d} \chi ;
$$

Integrating this from $\tau_{j-1}$ to $\tau_{j}$, we have

$$
\begin{array}{r}
\Delta I_{j} \equiv I\left(\tau_{j}\right)-I\left(\tau_{j-1}\right)=\int \mathrm{d} \chi \frac{F\left(\chi, \varepsilon \tau_{j}\right)}{i \chi}\left(e^{i \chi \tau_{j}}-e^{i \chi \tau_{j-1}}\right) \\
+\int_{\tau_{j-1}}^{\tau_{j}} \mathrm{~d} \tau \int \mathrm{d} \chi\left[F(\chi, \varepsilon \tau)-F\left(\chi, \varepsilon \tau_{j}\right)\right] e^{i \chi \tau} .
\end{array}
$$

In the latter integral, $\left|\varepsilon \tau-\varepsilon \tau_{j}\right| \leq \varepsilon^{1 / 2}$. According to estimates in Eq. (44)-(45),

$$
\left|\Delta I_{j}\right| \leq 2 M+\int_{\tau_{j-1}}^{\tau_{j}} L \varepsilon^{1 / 2} \mathrm{~d} \tau=2 M+L .
$$

Now, $|\Delta I| \leq \sum_{j=1}^{J}\left|\Delta I_{j}\right| \leq(2 M+L) J$, and we find Eq. (46).

\subsection{Interaction between three Rossby waves}

Now we estimate $\Delta I$ resulting from $i^{\mathrm{R}}$ in Eq. (41).

The periods of Rossby waves are $O\left(\beta^{-1}\right)$, and we introduce function

$\sigma(k)=-\frac{p}{k^{2}}, \quad$ so that $\quad \Omega(k)=\beta \sigma(k)$.
Rossby wave amplitude $\psi_{\boldsymbol{k}}$ evolves on the nonlinear timescale $O\left(\epsilon^{-1} \beta^{-1}\right)$ (recall the transformation in Eq. (39)). Therefore, to apply the lemma of Sect. 3.4, we assume

$\chi=-\left(\sigma_{1}+\sigma_{2}+\sigma_{3}\right), \quad \tau=\beta t, \quad \varepsilon=\epsilon$.

Now,

$$
\begin{aligned}
\frac{\mathrm{d} I^{\mathrm{R}}}{\mathrm{d} \tau} & =\frac{1}{\beta} \dot{I}^{\mathrm{R}}=\int F(\chi, \epsilon \tau) e^{i \chi \tau} \mathrm{d} \chi, \quad \text { with } \\
F & =\frac{1}{2 \beta} \int \mathrm{d}_{123} \delta\left(\sigma_{1}+\sigma_{2}+\sigma_{3}+\chi\right) \frac{X_{1}}{k_{1}^{2}} W_{123} \psi_{1} \psi_{2} \psi_{3} .
\end{aligned}
$$

We symmetrize the integral for $F$ with respect to permutations of indexes 1,2 , and 3 (this, in particular, gives an additional factor $1 / 3$ in front of the integral) and use Eq. (35) for the kernel $W$, taking into account that $\Delta_{1,2,3}$ is symmetric with respect to the cyclic permutations of its indexes

$$
\begin{aligned}
F= & \frac{1}{6 \beta} \int \mathrm{d}_{123} \delta\left(\boldsymbol{k}_{1}+\boldsymbol{k}_{2}+\boldsymbol{k}_{3}\right) \Delta_{1,2,3} \psi_{1} \psi_{2} \psi_{3} \\
& \delta\left(\sigma_{1}+\sigma_{2}+\sigma_{3}+\chi\right) \\
& {\left[\frac{X_{1}}{k_{1}^{2}}\left(k_{3}^{2}-k_{2}^{2}\right)+\frac{X_{2}}{k_{2}^{2}}\left(k_{1}^{2}-k_{3}^{2}\right)+\frac{X_{3}}{k_{3}^{2}}\left(k_{2}^{2}-k_{1}^{2}\right)\right] . }
\end{aligned}
$$

Now we will determine special functions $X(\boldsymbol{k})$ so that the integral (44) converges as $\chi \rightarrow 0$. Due to the dispersion relation, $k_{j}^{2}=-p_{j} / \sigma_{j}(j=1,2,3)$, so the square bracket in Eq. (49) equals

$$
\begin{aligned}
{[\ldots] } & =\frac{1}{\sigma_{1} \sigma_{2} \sigma_{3}}\left[\frac{p_{1} X_{1}}{k_{1}^{4}}\left(p_{3} \sigma_{2}-p_{2} \sigma_{3}\right)\right. \\
& \left.+\frac{p_{2} X_{2}}{k_{2}^{4}}\left(p_{1} \sigma_{3}-p_{3} \sigma_{1}\right)+\frac{p_{3} X_{3}}{k_{3}^{4}}\left(p_{2} \sigma_{1}-p_{1} \sigma_{2}\right)\right] .
\end{aligned}
$$

We have $p_{1}+p_{2}+p_{3}=0$ due to the delta function in Eq. (49); when $\chi=0$, then $\sigma_{1}+\sigma_{2}+\sigma_{3}=0$, and the three pairs of parentheses in Eq. (50) turn out equal. Now the square bracket in Eq. (49) is

$$
[\ldots]=\frac{p_{2} \sigma_{1}-p_{1} \sigma_{2}}{\sigma_{1} \sigma_{2} \sigma_{3}}\left[\frac{p_{1} X_{1}}{k_{1}^{4}}+\frac{p_{2} X_{2}}{k_{2}^{4}}+\frac{p_{3} X_{3}}{k_{3}^{4}}\right] ;
$$

it vanishes if the function

$\varphi(\boldsymbol{k})=\frac{p X(\boldsymbol{k})}{k^{4}}$

is conserved in resonant triad interactions

$$
\left.\begin{array}{l}
\boldsymbol{k}_{1}+\boldsymbol{k}_{2}+\boldsymbol{k}_{3}=0 \\
\Omega_{1}+\Omega_{2}+\Omega_{3}=0
\end{array}\right\} \Rightarrow \varphi_{1}+\varphi_{2}+\varphi_{3}=0
$$

Then the integral in Eq. (44) is convergent. Since $\psi_{\boldsymbol{k}}=$ $O(\epsilon \beta)$, the constants $M$ and $L$ are $\frac{1}{\beta} O\left(\epsilon^{3} \beta^{3}\right)=O\left(\epsilon^{3} \beta^{2}\right)$, and, according to the lemma, $\Delta I=O\left(\epsilon^{2.5} \beta^{2}\right)$. 
Remark. $\Omega(\boldsymbol{k})$ and $\varphi(\boldsymbol{k})$ are odd functions of the wave vector $\boldsymbol{k}$, so (implication (52)) is equivalent to the implication of Eqs. (12) $\Rightarrow$ (11).

There are five possible functions $\varphi(\boldsymbol{k})$ that satisfy Eq. (52). Three of them are obvious and common:

$\varphi(\boldsymbol{k})=\Omega(\boldsymbol{k}), \quad \varphi(\boldsymbol{k})=p, \quad \varphi(\boldsymbol{k})=q$.

The other two (Balk, 1991) are specific for short Rossby waves:

$\varphi(\boldsymbol{k})=\xi^{\text {short }}(\boldsymbol{k}) \equiv \frac{p q}{k^{4}} ;$

$\varphi(\boldsymbol{k})=\eta^{\text {short }}(\boldsymbol{k}) \equiv \frac{p^{3}\left(p^{2}+5 q^{2}\right)}{k^{10}}$.

Similar to Balk et al. (2011), we discard the third and the fourth of them as unphysical: the third gives singular $X(\boldsymbol{k})=$ $q k^{4} / p$ (on the line $p=0$ ), and the fourth is even, giving odd $X(\boldsymbol{k})$, which contradicts to the bracketed condition in Eq. (37). The first function in Eq. (53) corresponds to the energy of the quasi-geostrophic mode; the second corresponds to the zonal momentum (which is the enstrophy).

The fifth function (Eq. 55) gives the extra invariant (cf. Eq. 18).

\subsection{Interaction between one Rossby wave and two inertia waves}

Now, we estimate the variation $\Delta I$ resulting from $\dot{I}^{\mathrm{i}}$ in Eq. (41).

The inertia wave amplitude $A_{k}(t)$ evolves faster than the Rossby wave amplitude $\psi_{k}(t)$. The latter evolves on the nonlinear timescale $O\left(\epsilon^{-1} \beta^{-1}\right)$. The former have linear response to the Rossby waves. Recall that we used normal modes for $\beta=0$, so $A_{k}$ and $\psi_{k}$ are independent in linearized dynamics if $\beta=0$ only. If $\beta \neq 0$, the amplitude $A_{\boldsymbol{k}}(t)$ evolves on timescale $O\left(\beta^{-1}\right)$. Therefore, we use the lemma of Sect. 3.4 with

$\chi=-\left(\omega_{2}+\omega_{3}\right), \quad \tau=t, \quad \varepsilon=\beta$.

Now,

$\frac{\mathrm{d} I^{\mathrm{i}}}{\mathrm{d} t}=\int F(\chi, \beta t) e^{i \chi t} \mathrm{~d} \chi$,

where

$F=\frac{1}{2} \int \mathrm{d}_{123} \sum_{m_{2}, m_{3}} \delta\left(\omega_{2}+\omega_{3}+\chi\right) \frac{X_{1}}{k_{1}^{2}} V_{123} \Psi_{1} A_{2} A_{3}$.

To apply the lemma, we show that the integral in Eq. (44) converges as $\chi \rightarrow 0$. Indeed, when $\chi=0$, then $\omega_{2}+\omega_{3}=0$, which implies $\left|\omega_{2}\right|=\left|\omega_{3}\right|$. Because of Kronecker deltas in Eq. (36), we have $\left|m_{2}\right|=\left|m_{3}\right|$. Therefore, - by the dispersion relation in Eq. (31) $-K_{2}=K_{3}$, and $k_{2}=k_{3}$. Now,

$\boldsymbol{k}_{\mathbf{2}}=-\boldsymbol{k}_{1}-\boldsymbol{k}_{3} \Rightarrow k_{2}^{2}=k_{1}^{2}+k_{3}^{2}+2 \boldsymbol{k}_{1} \cdot \boldsymbol{k}_{3}$,

$\boldsymbol{k}_{\mathbf{3}}=-\boldsymbol{k}_{1}-\boldsymbol{k}_{2} \Rightarrow k_{3}^{2}=k_{1}^{2}+k_{2}^{2}+2 \boldsymbol{k}_{1} \cdot \boldsymbol{k}_{2}$.
Thus $\boldsymbol{k}_{1} \cdot \boldsymbol{k}_{3}=\boldsymbol{k}_{1} \cdot \boldsymbol{k}_{2}$, and in the square bracket of Eq. (36), the four pairs of parentheses are pairwise identical. Therefore, $V_{1,2,3}=0$.

The wave amplitudes $\Psi$ and $A$ are $O(\epsilon \beta)$, and, therefore, the constants $M$ and $L$ are $O\left(\epsilon^{3} \beta^{3}\right)$. Now, according to the lemma, $\Delta I=O\left(\epsilon^{3} \beta^{2.5}\right)$ on any time interval of length $\beta^{-1}$. We can split the time interval $0 \leq t \leq \epsilon^{-1} \beta^{-1}$ into subintervals of length $O\left(\beta^{-1}\right)$; there are $O\left(\epsilon^{-1}\right)$ of them. Thus, $\Delta I=\frac{1}{\epsilon} O\left(\epsilon^{3} \beta^{2.5}\right)$ on time interval $0 \leq t \leq \epsilon^{-1} \beta^{-1}$.

Combining this estimate and the estimate found in the previous Sect. 3.5, we find Eq. (27).

Remark. The estimate in Eq. (27) holds for any solution of the 3-D layer dynamics in Eq. (22)-(24). If the wave turbulence were considered, the estimate would hold for any realization, even the least probable; on average (or for a typical realization) the estimate is stronger.

\section{Emergence of zonal jets}

\subsection{Invariants}

According to the expansion in Eq. (32), the vertical vorticity in Eq. (26) is

$\zeta(x, y, t)=-\int k^{2} \Psi_{\boldsymbol{k}}(t) e^{i(p x+q y)} \mathrm{d} \boldsymbol{k}$

(since the cosines integrate to zero); in the Fourier representation, Eq. (57) means $\zeta_{k}=-k^{2} \Psi_{k}$. So, we can express integral in Eq. (37) in terms of $\zeta_{k}$ :

$I=\frac{1}{2} \int \frac{1}{p} \varphi(\boldsymbol{k})\left|\zeta_{\boldsymbol{k}}\right|^{2} d \boldsymbol{k}$.

Thus, we have three invariants:

1. The energy of the quasi-geostrophic mode (the energy of inertia waves is separate)

$E=\frac{1}{2} \int \frac{1}{k^{2}}\left|\zeta_{k}\right|^{2} \mathrm{~d} \boldsymbol{k}$

2. The enstrophy

Enstrophy $=\frac{1}{2} \int\left|\zeta_{\boldsymbol{k}}\right|^{2} \mathrm{~d} \boldsymbol{k}$.

3. The extra invariant

$I=\frac{1}{2} \int \frac{1}{p} \eta^{\text {short }}(\boldsymbol{k})\left|\zeta_{\boldsymbol{k}}\right|^{2} \mathrm{~d} \boldsymbol{k}$

with $\eta^{\text {short }}(\boldsymbol{k})$ given in Eq. (55). Though the function in Eq. (55) has non-integrable singularity at $k=0$, the energy spectrum usually vanishes at $k \rightarrow 0$, and the integral in Eq. (61) converges. The expression in Eq. (61) is the same as Eq. (25). 


\subsection{Anisotropic inverse cascade}

In rapidly rotating 3-D fluids, the energy from inertia waves is transferred towards the quasi-geostrophic mode (Smith and Waleffe, 1999; Cambon et al., 2004; Staplehurst et al., 2008; Duran-Matute et al., 2013).

The conservation of the energy in Eq. (59) and the enstrophy in Eq. (60) implies the inverse cascade of the twodimensional quasi-geostrophic energy (Rhines, 1975; Pedlosky, 1987; Vallis, 2006). This is because the energy spectrum spreads in the Fourier space (unless the initial conditions are very specially arranged); the spreading happens due to (Rhines, 1975) the decay instability (when a wave decays into the other two waves of a resonant triad), as well as due to the resonant generation of higher harmonics.

We assume that the inverse energy cascade takes place. Then the presence of the third, extra, invariant in Eq. (61) implies the energy transfer towards large-scale zonal flow, not just to large scales in general, but specifically into the region of the $\boldsymbol{k}$ plane around the $q$ axis. This is the only way to keep the extra invariant approximately constant.

Indeed, let $E_{k}=\left|\zeta_{k}\right|^{2} / k^{2}$ be the energy spectrum of the quasi-geostrophic mode. According to Eqs. (59) and (61), the dynamics preserves integrals

$E=\frac{1}{2} \int E_{k} \mathrm{~d} \boldsymbol{k} \quad$ and $\quad I=\frac{1}{2} \int \phi(\boldsymbol{k}) E_{\boldsymbol{k}} \mathrm{d} \boldsymbol{k}$,

where

$\phi(\boldsymbol{k})=\frac{\eta^{\text {short }}(\boldsymbol{k})}{-\Omega(\boldsymbol{k}) / \beta}=\frac{p^{2}\left(p^{2}+5 q^{2}\right)}{k^{8}}$

is shown in Fig. 1. The ratio $\phi$ is small for large $k$, and large near the origin, except for the vicinity of the $q$ axis. Therefore, the energy transferring from large $k$ towards the origin - via the inverse cascade - must accumulate near the $q$ axis (which corresponds to zonal jets). Such an anisotropic inverse cascade is similar to the one in geostrophic dynamics (see Balk, 2005, for precise estimates). The reasoning for the generation of zonal flow tacitly assumes that there is a source of small-scale energy. We see that the formation of zonal jets in 3-D dynamics is enhanced: because of the two-dimensionalization, the energy of 3-D inertia waves also transfers into zonal jets.

\section{Conclusion}

The system in Eq. (22) is known to conserve the energy and helicity. We have shown that, in the presence of the beta effect $\left(\beta \equiv f^{\prime}(y) \neq 0\right)$, there are three adiabatic-type invariants: the energy (Eq. 59), the enstrophy (Eq. 60), and the extra invariant (Eq. 61), all being based on the Rossby waves.

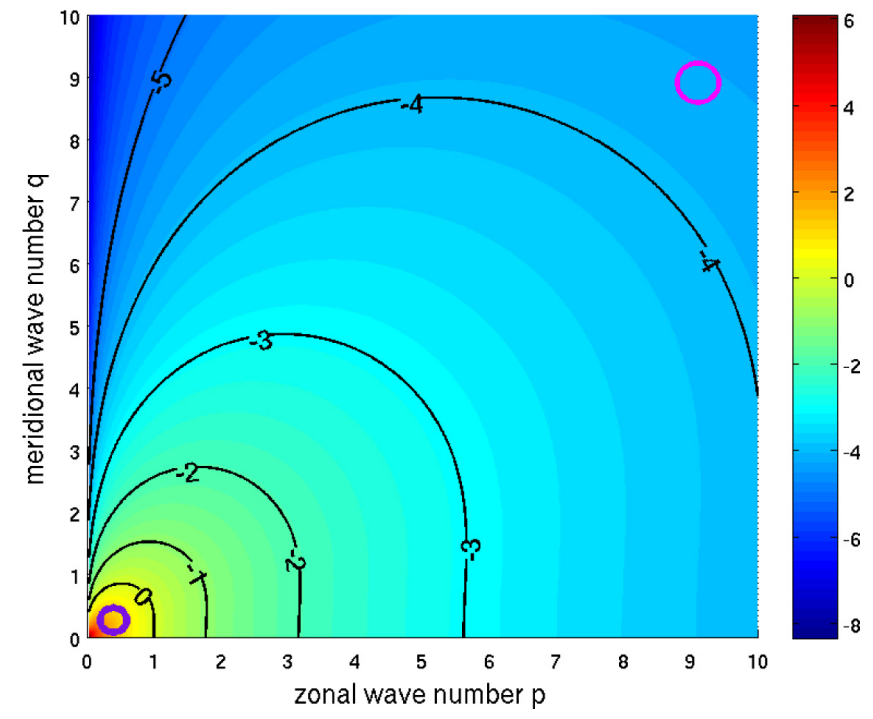

Fig. 1. The values of the ratio $\phi=\frac{\eta^{\text {short }}}{-\Omega / \beta}$ (in logarithmic scale) as a function of the wave vector $\boldsymbol{k}=(p, q)$. The numbers of the color bar are the values of $\log _{10}(\phi)$. The black curves are the level lines $\phi(\boldsymbol{k})=10^{n}$ for six integers $n=0,-1,-2,-3,-4,-5$. All the contour lines pass through the origin tangent to the $q$ axis. The figure shows that the inverse cascade transfers energy from smallscale eddies to large-scale zonal jets. Indeed, let us pose a question: is it possible that the energy from the area marked by the pink circle (in the upper right corner) - via the inverse cascade - ends up in the purple circle near the origin? This is clearly impossible when both integrals $\int E_{\boldsymbol{k}} \mathrm{d} \boldsymbol{k}$ and $\int \phi(\boldsymbol{k}) E_{\boldsymbol{k}} \mathrm{d} \boldsymbol{k}$ are preserved. If such transfer were to occur, it would lead to a significant increase of the extra invariant: the value of the ratio $\phi$ in the purple circle is more than six orders of magnitude bigger than its value in the pink circle. The only possibility for the inverse cascade is that the energy should end up near the $q$ axis where the ratio $\phi$ is also small, similar to its values at large wave numbers (away from the $q$ axis), so that the energy and extra invariant in Eq. (62) can be both conserved. The energy concentration near the $q$ axis means the fluid should have velocity mostly parallel to the $x$ axis, which is zonal flow.

The presence of these invariants - together with the twodimensionalization - implies the transfer of energy towards zonal jets (Sect. 4.2).

We have also summarized the unique conservation properties of the geophysical fluid dynamics (with the beta effect) that allow for the presence of the Rossby wave extra invariant in the various systems (Sect. 2). It seems possible to extend the extra invariant to the stratified fluid dynamics that involves several Rossby modes with different Rossby radii of deformation (e.g., to the systems with several shallow layers). This is plausible because the coupling coefficients of resonant triad interactions between the barotropic mode and the other Rossby modes vanish, provided the densities of the layers are sufficiently close (Soomere, 2003). 
Acknowledgements. I wish to thank L. Smith for her suggestion to consider 3-D dynamics. As well, I am grateful to E. Ferapontov for introducing to me Boltzmann's problem of collision invariants (Sect. 1.1) many years ago and to P. Weichman for the valuable possibility to discuss the extra invariant. I am also thankful for discussions of this research at the workshop "Nonlinear processes in atmospheric and oceanic flows", the Instituto de Ciencias Matemáticas (ICMAT), Madrid, Spain, July 2012. The provided support is gratefully acknowledged. The publication of the present paper is funded by the ICMAT Severo Ochoa project SEV-2011-0087. Likewise, I am grateful for discussions during the program Stochastic Flows and Climate Modeling, Aspen Center for Physics, Colorado, USA, June 2012. The present work was supported in part by the National Science Foundation under grant no. PHYS-1066293 and the hospitality of the Aspen Center.

Edited by: E. Hernández-García

Reviewed by: four anonymous referees

\section{References}

Balk, A. M.: A new invariant for Rossby wave systems, Phys. Lett. A, 155, 20-24, 1991.

Balk, A. M.: New conservation laws for the interaction of nonlinear waves, SIAM Rev., 39, 68-94, 1997.

Balk, A. M.: Angular distribution of Rossby wave energy, Phys. Lett. A, 345, 154-160, 2005.

Balk, A. M. and Ferapontov, E. V.: Invariants of 4-wave interactions, Physica D, 65, 274-288, 1993.

Balk, A. M. and Ferapontov, E. V.: Invariants of wave systems and web geometry, in: Nonlinear waves and weak turbulence, edited by: Zakharov, V. E., T. Am. Math. Soc., Ser. 2, vol. 182, 1-30, 1998.

Balk, A. M. and van Heerden, F.: Conservation style of the extra invariant for Rossby waves, Physica D, 223, 109-120, 2006.

Balk, A. M., Nazarenko, S. V., and Zakharov, V. E.: New invariant for drift turbulence, Phys. Lett. A, 152, 276-280, 1991.

Balk, A. M., van Heerden, F., and Weichman, P. B.: Rotating shallow water dynamics: Extra invariant and the formation of zonal jets, Phys. Rev. E, 83, 046320, doi:10.1103/PhysRevE.83.046320, 2011.

Blaschke, W.: Topological differential geometry, Chicago University Press, USA, 1932

Boltzmann, L.: Über das warmegleichgewicht von gasen, auf welche äußere kräfte wirken, Wiener Berichte, 72, 427-457, 1875 (in German).

Cambon, C., Rubinstein, R., and Godeferd, F. S.: Advances in wave turbulence: rapidly rotating flows, New J. Phys., 6, 73, 29 pp., 2004.

Duran-Matute, M., Flór, J.-B., Godeferd, F. S., and JauseLabert, C.: Turbulence and columnar vortex formation through inertial-wave focusing, Phys. Rev. E, 87, 041001(R), doi:10.1103/PhysRevE.87.041001, 2013.
Ferapontov, E. V.: Web geometry and mathematical physics, in: Geometry and Algebra of Multidimensional Three-Webs, authored by: Akivis, M. A. and Shelekhov, A. M., Kluwer Academic Publishers, Norwell, MA, 310-323, 1992.

Gill, A. E.: Atmosphere-Ocean Dynamics, Academic Press, New York, 1982.

Greenspan, H. P.: On the nonlinear interaction of inertial modes, J. Fluid Mech., 36, 257-264, 1969.

Landau, L. D. and Lifshitz, E. M.: Mechanics, Course of theoretical physics, v. 1, 3rd Edn., Butterworth-Heinemann, New York, 1976.

Landau, L. D. and Lifshitz, E. M.: Fluid Mechanics, Course of theoretical physics, v. 6, 2nd Edn., Butterworth-Heinemann, New York, 1987.

Manz, P., Xu, G. S., Wan, B. N., Wang, H. Q., Guo, H. Y., Cziegler, I., Fedorczak, N., Holland, C., Muller, S. H., Thakur, S. C., Xu, M., Miki, K., Diamond, P. H., and Tynan, G. R.: Zonal flow triggers the L-H transition in the Experimental Advanced Superconducting Tokamak, Phys. Plasmas, 19, 072311 , doi:10.1063/1.4737612, 2012.

Maximenko, N., Bang, B., and Sasaki, H.: Observational evidence of alternating zonal jets in the world ocean, Geophys. Res. Lett., 32, L12607, doi:10.1029/2005GL022728, 2005.

Nazarenko, S. and Quinn, B.: Triple Cascade Behavior in Quasigeostrophic and Drift Turbulence and Generation of Zonal Jets, Phys. Rev. Lett., 103, 118501, doi:10.1103/PhysRevLett.103.118501, 2009.

Pedlosky, J.: Geophysical Fluid Dynamics, Springer, New York, 1987.

Rhines, P. B.: Waves and turbulence on a beta plane, J. Fluid Mech. 69, 417-443, 1975.

Sercignani, C.: Are there more than 5 linearly-independent collision invariants for the Boltzmann equation?, J. Stat. Phys., 58, 817823, 1990.

Smith, L. M. and Waleffe, F.: Transfer of energy to two-dimensional large scales in forced, rotating three-dimensional turbulence, Phys. Fluids, 11, 1608-1622, 1999.

Soomere, T.: Coupling coefficients and kinetic equation for Rossby waves in multi-layer ocean, Nonlin. Processes Geophys., 10, 385-396, doi:10.5194/npg-10-385-2003, 2003.

Staplehurst, P. J., Davidson, P. A., and Dalziel, S. B.: Structure formation in homogeneous freely decaying rotating turbulence, J. Fluid Mech., 598, 81-105, 2008.

Vallis, G. K.: Atmospheric and Oceanic Fluid Dynamics, Cambridge University Press, Cambridge, UK, 2006.

Zakharov, V. E. and Schulman, E. I.: Degenerative dispersion laws, motion invariants, and kinetic equations, Physica D, 10, 192 202, 1980.

Zakharov, V. E. and Schulman, E. I.: On additional motion invariants of classical Hamiltonian wave systems, Physica D, 29, 283 $320,1988$. 